\title{
EFFETS DU CONDITIONNEMENT EN JOURS LONGS À LA FIN DU CYCLE REPRODUCTEUR SUR LA PÉRIODE D'OVULATION ET SUR LES SÉCRÉTIONS GONADOTROPES CHEZ L'OMBLE CHEVALIER (SALVELINUS ALPINUS).
}

\author{
C. GILLET (1), I. RIDEAU $(1,2)$ et B. BRETON (2)
}

(1) Station d'Hydrobiologie Lacustre, 75 avenue de Corzent, 74203 Thonon-les-Bains Cedex, France.

(2) IFR 42, Laboratoire de Physiologie des Poissons, INRA, Campus de Beaulieu, 35042 Rennes Cedex, France.

\section{RÉSUMÉ}

Les ombles chevaliers originaires du Léman se reproduisent à la fin de l'automne. En élevage, il est intéressant de retarder de quelques mois la période des ovulations car ce poisson a besoin d'une température inférieure à $6^{\circ} \mathrm{C}$ pendant l'ovulation. II est plus facile de fournir une eau à cette température aux géniteurs retardés qui ovulent en hiver qu'à ceux qui ovulent naturellement en automne.

Le conditionnement des ombles chevaliers en jours longs $(17 \mathrm{~L}-7 \mathrm{~N})$ pendant l'automne permet de retarder de plusieurs mois la période des ovulations. Cette méthode a l'inconvénient de provoquer un étalement des ovulations sur plus de 3 mois. Le reconditionnement des géniteurs en jours courts en décembre supprime ce problème. L'efficacité du traitement en jours longs ne semble pas dépendre de la durée de son application pourvu que celle-ci dépasse 1,5 mois.

Chez les femelles conditionnées en jours longs en automne et en hiver, les concentrations en gonadotropine plasmatique (GTH II) sont très faibles. Le niveau de la GTH II plasmatique augmente trois semaines après un transfert en jours courts en janvier. Les concentrations de la GTH II plasmatique au cours de l'ovulation sont significativement plus faibles chez les femelles conditionnées en jours longs en janvier que chez les animaux reconditionnés en jours courts. A ce stade du cycle reproducteur, la GTH I plasmatique reste toujours à un niveau très faible, quel que soit le régime photopériodique. La pose d'implant de mélatonine chez les poissons ne modifie pas les sécrétions gonadotropes en jours longs comme en jours courts.

La réceptivité hypophysaire des femelles à une stimulation de la sécrétion gonadotrope par une injection de GnRH n'est pas modifiée par le conditionnement des poissons en jours longs. Aucun effet du pimozide sur la sécrétion de la GTH II n'a pu être mis en évidence chez les géniteurs conditionnés en jours longs. Ces résultats permettent de supposer que l'action inhibitrice des jours longs sur l'ovulation n'est pas la conséquence d'un blocage de type dopaminergique de la sécrétion gonadotrope. Ce type d'inhibition mettrait en oeuvre des mécanismes différents de ceux impliqués dans le blocage de l'ovulation par des températures élevées. L'effet inhibiteur des jours longs à ce stade du cycle reproducteur passe par une diminution de la sécrétion gonadotrope de la GTH II, probablement en relation avec des modifications du contrôle hypothalamique de la sécrétion de la gonadotropine hypophysaire (GTH II). 


\title{
EFFECTS OF ACCLIMATIZATION IN LONG DAYS AT THE END OF THE REPRODUCTIVE CYCLE ON THE TIMING OF OVULATION AND ON GONADOTROPIN SECRETIONS IN ARCTIC CHARR (SALVELINUS ALPINUS).
}

\begin{abstract}
Arctic charr originated from Lake Geneva spawn at the end of autumn. In hatchery, it is advantageous to delay ovulation from several months because Arctic charr require temperatures below $6^{\circ} \mathrm{C}$ for ovulation and it is easier to supply cold water to broodstock in winter than in fall.
\end{abstract}

The acclimatization of Arctic charr in long days during fall delayed ovulation time of several months. This method had the disadvantage to extend the ovulation time over more than three months. This problem was suppressed by transferring again the broodstock under short days during December. The efficiency of the long day treatment to delay ovulation did not depend on its length when the treatment lasted longer than 1.5 months.

In females acclimatized in long days during fall and winter, plasma gonadotropin levels (GTH II) were always very low. After a transfer from long days to short days in January, plasma GTH II levels raised within three weeks. Plasma GTH II levels were significantly lower during the ovulation period in females maintained under long days than in females transferred in short days. At this stage of reproductive cycle, plasma GTH I levels were always very low, whatever the photoperiod regime. A melatonin treatment given by intraperitoneal implants did not modify gonadotropin secretions in both long day or short day conditions.

The pituitary responsiveness of females to a stimulation of gonadotropin secretion by $\mathrm{GnRH}$ injections did not differ between fish reared in long days or in short days. Pimozide treatment did not modify the pituitary responsiveness to $\mathrm{GnRH}$ in females acclimatized in long day photoperiod. As a whole, the results indicated that the inhibitory effect of long days on GTH II secretion did not involve a blockade by dopamine contrary to the inhibitory effect of warm temperatures on the same phenomenon. The inhibition of GTH II secretion at final stages of the reproductive cycles probably involved changes in the hypothalamic control of GTH II secretion.

\section{INTRODUCTION}

Chez les salmonidés, de nombreuses recherches ont permis d'établir avec certitude que la photopériode exerce un contrôle déterminant sur le caractère saisonnier de la reproduction. Les applications de ces recherches ont abouti à la réalisation des manipulations photopériodiques relativement simples permettant d'étaler la production en oeufs (BRETON et al., 1983 ; BROMAGE et DUSTON, 1986). Chez l'omble chevalier, deux facteurs externes, la photopériode et la température exercent un contrôle direct sur le déroulement du cycle reproducteur. Une inhibition se met en place lorsque la température de l'eau atteint ou dépasse $10^{\circ} \mathrm{C}$ (GILLET, 1991). A la fin de l'automne, période de reproduction de l'omble en France, il est souvent difficile de procurer aux géniteurs de l'eau vers $5^{\circ} \mathrm{C}$, température nécessaire pour l'obtention d'ovules présentant une bonne fécondabilité. En retardant de deux mois environ, la ponte par manipulation photopériodique, il est plus facile de disposer d'eau froide, ce qui se traduit par une amélioration de la qualité des gamètes (GILLET, 1994). Cependant le retard de ponte, obtenu en conditionnant les géniteurs de salmonidé en jours longs en fin de cycle, s'accompagne d'un étalement important des ovulations. Ce phénomène entraîne une gestion difficile du stock de géniteurs (MAISSE et BRETON, 1996). 
Les expérimentations présentées dans cet article visent à optimiser les manipulations photopériodiques destinées à retarder la ponte des ombles chevaliers. Dans ce travail, nous avons également cherché à savoir si les effets de la photopériode et de la température se réalisaient à travers des modifications des sécrétions gonadotropes, surtout celle de la GTH II plus particulièrement impliquée dans le contrôle endocrinien de la maturation ovocytaire. Nous avons aussi étudié les effets de ces facteurs sur la possibilité de stimuler la fonction gonadotrope hypophysaire par la gonadolibérine $(\mathrm{GnRH})$, indice d'une action sur l'activation de la fonction gonadotrope centrale. La mélatonine joue un rôle fondamental dans le transfert de la stimulation photopériodique vers les centres nerveux qui contrôlent les sécrétions gonadotropes chez les vertébrés supérieurs. Chez les ovins en particulier, l'administration de mélatonine sous forme d'implant reproduit les effets d'un conditionnement en jours longs (MALPAUX et al., 1996). Nous avons également testé l'effet des implants de mélatonine chez l'omble chevalier. La conformité des résultats de cette étude avec l'hypothèse selon laquelle la photopériode agirait en entraînant un rythme interne (DUSTON et BROMAGE, 1988) est examinée.

\section{MATÉRIELS ET MÉTHODES}

\section{Procédures d'élevage}

Les géniteurs âgés de 3 ans sont élevés dans des bacs de 4 et $12 \mathrm{~m}^{2}$, alimentés par de l'eau pompée à $50 \mathrm{~m}$ de profondeur dans le Léman dont la température fluctue entre $9^{\circ} \mathrm{C}$ en été et $6^{\circ} \mathrm{C}$ à la fin de l'hiver (fig. 1, I). La nourriture composée de granulés trouvit est distribuée en continu 8 heures par jour à $1 \%$ de la biomasse. Un système de réfrigération permet de stabiliser la température de certains bacs à $5^{\circ} \mathrm{C}$ et une source à température constante permet de maintenir $10^{\circ} \mathrm{C}$ en hiver dans d'autres bacs. Chaque mois depuis mai jusqu'en décembre, 10 femelles sont sacrifiées pour calculer leur indice gonadosomatique (RGS $=$ poids des ovaires/poids du corps ${ }^{*} 100$ ).

\section{2. Étude de différents types de conditionnement en jours longs en fin de gamétogenèse ou en période d'ovulation}

Les géniteurs sont élevés jusqu'en août en photopériode naturelle. A partir du 28/08, des groupes d'une vingtaine de femelles sont transférés sous 17 heures d'éclairement journalier $(17 \mathrm{~L}-7 \mathrm{~N}=\mathrm{JL}$ ) à différentes dates. Deux groupes sont maintenus en JL pendant toute la durée des ovulations tandis que deux autres groupes sont retransférés en photopériode naturelle avant les premières ovulations. Les témoins sont élevés toute l'année en photopériode naturelie (fig. 1, II). Dans une deuxième série d'expérimentations, quatre groupes de femelles sont acclimatés en JL en août, septembre, octobre et novembre puis ils sont tous retransférés en photopériode naturelle le 16/12 (fig. 1, III). Tous les groupes sont contrôlés une fois par semaine pour détecter les ovulations.

3. Étude des sécrétions gonadotropes et de la réceptivité hypophysaire au GnRH chez des femelles acclimatées en jours longs et en jours courts à 5 et $10^{\circ} \mathrm{C}$ en janvier

Des géniteurs sont acclimatés en septembre en $\mathrm{JL}$ et sont maintenus dans ces conditions jusqu'au 23/01. A cette date, deux groupes de 30 femelles sont acclimatés en jours courts $(7 \mathrm{~L}-17 \mathrm{~N}=\mathrm{JC}$ ) et deux autres groupes sont gardés en JL. Sous chaque photopériode, un groupe est acclimaté à $5^{\circ} \mathrm{C}$ et l'autre à $10^{\circ} \mathrm{C}$ (fig. 2 ). Dans chacun des quatre groupes ainsi constitués $\left(\mathrm{JC} 5^{\circ} \mathrm{C}, \mathrm{JC} 10^{\circ} \mathrm{C}, \mathrm{JL} 5^{\circ} \mathrm{C}\right.$ et $\mathrm{JL} 10^{\circ} \mathrm{C}$ ), cinq femelles reçoivent un implant de mélatonine dans l'abdomen $(5 \mathrm{mg} / \mathrm{kg}$, matrice en silicone). Dans tous les groupes, vingt femelles (dont les cinq traitées à la mélatonine) reçoivent une micromarque oculaire numérotée. Le 23/01, le 30/01, le 7/02 et le 14/02, les 20 poissons 


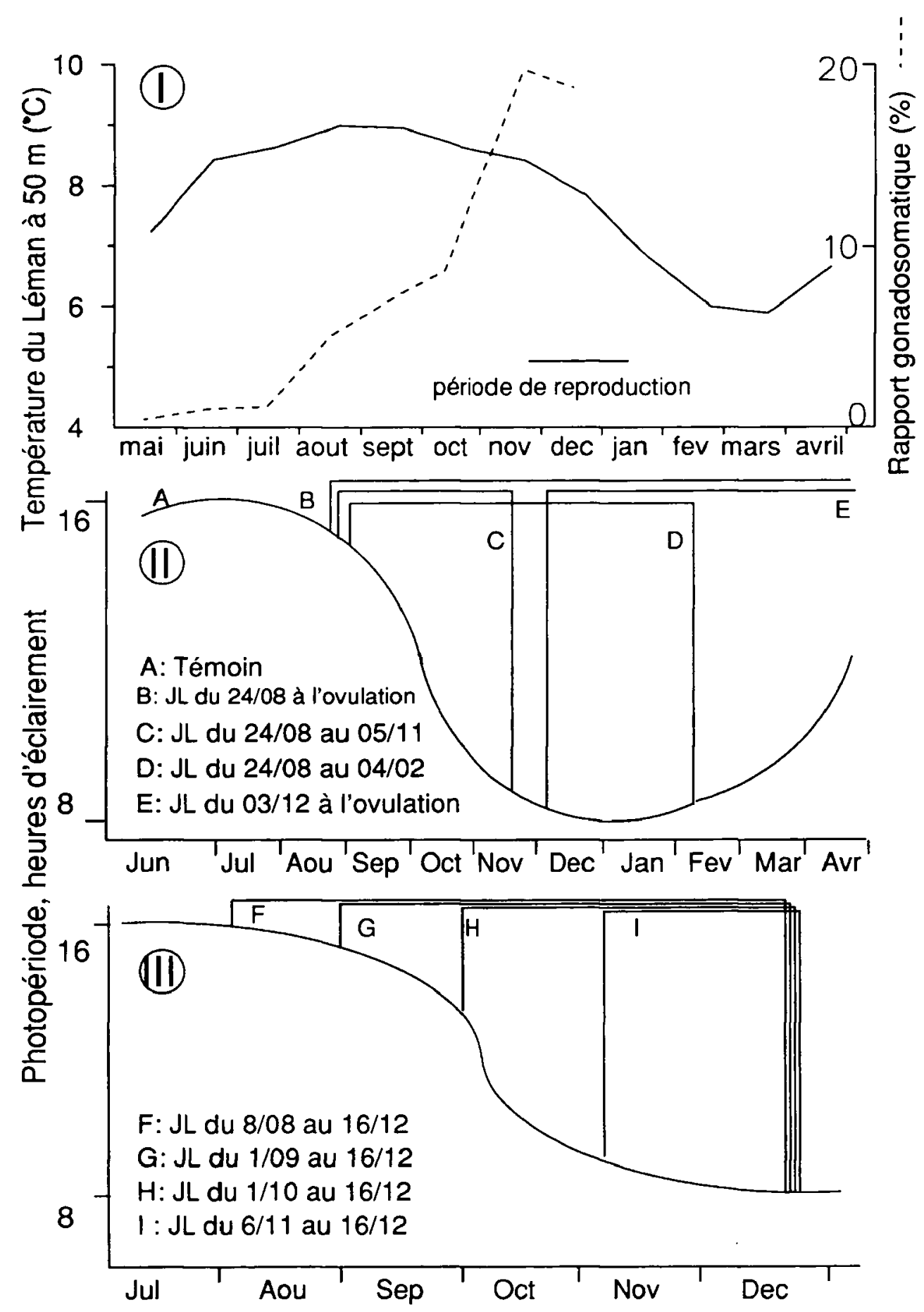

Figure 1

I - Évolutions de la température mensuelle de l'eau du Léman pompée à $50 \mathrm{~m}$ et du rapport gonadosomatique des ombles chevaliers en élevage. II et III - Différents traitements photopériodiques en jours longs appliqués aux ombles chevaliers pour retarder les ovulations.

Figure 1

I - Evolution in monthly temperature of Lake Geneva water pumped at a $50 \mathrm{~m}$ depth and in the gonadosomatic index of Arctic charr broodstock. II and III - Different long day treatments tested in Arctic charr to delay ovulation. 
marqués de chaque groupe sont anesthésiés avec du Marinil $(5 \mathrm{mg} / \mathrm{l})$. Des échantillons sanguins sont prélevés par ponction caudale et les ovulations sont enregistrées. Le 28/02, dans chacun des quatre groupes, 15 femelles non ovulées sont sélectionnées pour tester leur réceptivité hypophysaire à une stimulation par du $\mathrm{GnRHa} .5$ femelles reçoivent une injection intrapéritonéale de $D$ Arg ${ }^{6} s \mathrm{G} R H(20 \mu \mathrm{g} / \mathrm{kg})$ diluée dans du sérum physiologique, 5 autres femelles reçoivent la mème dose de $\mathrm{GnRH}$ associée à du pimozide $(5 \mathrm{mg} / \mathrm{kg}$ ) et 5 femelles témoins reçoivent du sérum physiologique. Des échantillons sanguins sont prélevés $0,5,10$ et 24 heures après le traitement.

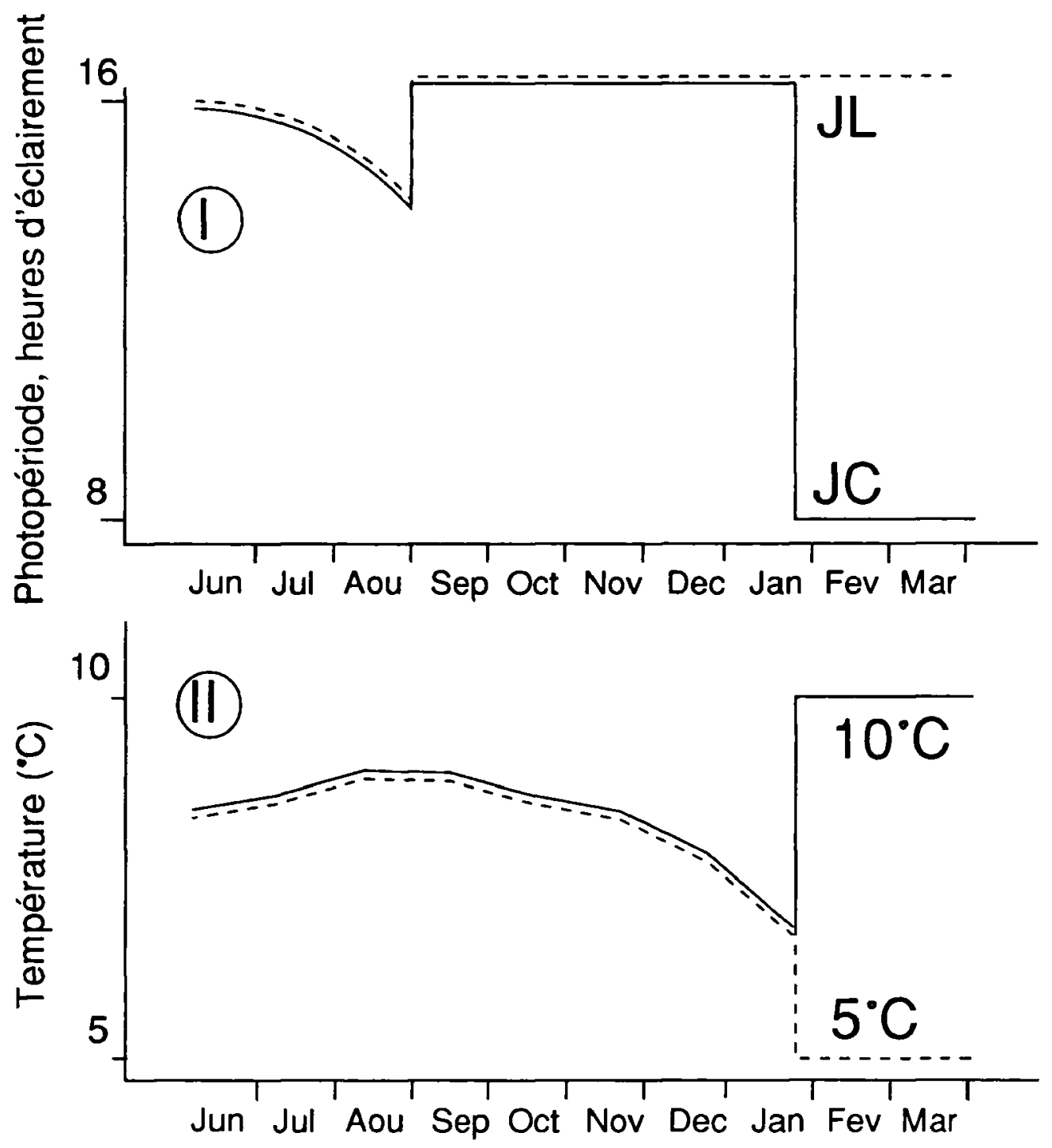

Figure 2

Régimes photopériodiques (I) et thermiques (II) auxquels sont soumis les ombles chevaliers utilisés en janvier 1996 dans les expérimentations portant sur les sécrétions gonadotropes et la réceptivité hypophysaire au GnRH. 4 groupes de 20 femelles sont constitués le $23 / 01: 5^{\circ} \mathrm{C} \mathrm{JC}, 5^{\circ} \mathrm{C} \mathrm{JL}, 10^{\circ} \mathrm{C} \mathrm{JC}$ et $10^{\circ} \mathrm{C} \mathrm{JL}$.

Figure 2

Photoperiodic (I) and thermal (II) regimes which were applied to Arctic charr on January 1996 to investigate gonadotropin secretions and pituitary responsiveness to GnRH. 4 groups with 20 females were made on 23 January : $5^{\circ} \mathrm{C}$, short days ; $10^{\circ} \mathrm{C}$, short days $; 5^{\circ} \mathrm{C}$, long days and $10^{\circ} \mathrm{C}$, long days. 
Le sang est centrifugé (15 mn, $5000 \mathrm{~g})$ et le plasma est congelé. Après décongélation, les hormones gonadotropes plasmatiques (GTH I et GTH II) sont dosées par radio-immunologie selon la méthode de GOVORUM et BRETON (1997). Les moyennes sont comparées par les tests non paramétriques des rangs, de Kruskal et Wallis et de Friedman.

\section{RÉSULTATS}

\section{Rythme des ovulations chez les poissons conditionnés en JL en fin de cycle reproducteur}

Les ovaires des poissons témoins sont relativement peu développés en août (RGS $=5 \%$ ). Ils atteignent leur développement maximum en novembre (RGS $=20 \%$ ) (fig. 1,1 ). Les ovulations se produisent à partir de début décembre jusqu'à la mi-janvier (fig. 3). La période d'ovulation de tous les groupes acclimatés en JL en fin de cycle est significativement plus tardive que celle des témoins $(P<0.01)$. L'étalement de la période d'ovulation est plus important chez les poissons maintenus en JL pendant l'ovulation que chez ceux qui ovulent en photopériode naturelle, quelle que soit la date d'acclimatation en $\mathrm{JL}$. Cela se traduit par une variance des dates d'ovulation significativement plus importante chez les poissons acclimatés en JL que chez ceux qui ovulent en photopériode naturelle $(P<0.05)$. La durée de la période d'ovulation est comparable à celle des témoins lorsque les poissons sont retransférés en jours courts avant l'ovulation, quelle que soit la date du transfert (fig. 3). Les rythmes d'ovulation des poissons acclimatés en JL depuis août, septembre ou octobre jusqu'au 16/12 présentent sensiblement le même retard par rapport aux témoins $(P<0.01)$ tandis que ceux acclimatés en $\mathrm{JL}$ de novembre au $16 / 12$ ovulent en moyenne 15 jours plus tôt (fig. 4).

\section{Sécrétions gonadotropes et réceptivité hypophysaire au GnRH chez les femelles acclimatées en $\mathrm{JC}$ et en $\mathrm{JL}$ à 5 et $10^{\circ} \mathrm{C}$}

Les teneurs de la GTH II plasmatique sont très faibles le 23/01, chez les femelles conditionnées depuis cinq mois en $\mathrm{JL}$. Elles n'augmentent significativement $(P<0.01)$ par la suite que chez les animaux transférés à $5^{\circ} \mathrm{C}$. Après quatre semaines d'acclimatation à cette température, les niveaux en GTH II plasmatique sont significativement plus élevés chez les animaux transférés en JC que chez ceux maintenus en JL (fig. 5). L'augmentation de la GTH II est liée en partie au moins à la présence de femelles ovulées : 11 femelles ovulent sur les 19 présentes en JC et 5/20 en JL entre le 23/01 et le 28/02. Aucune femelle n'avait ovulé avant le 23/01. Le test de la probabilité exacte de Fisher permet de conclure que les proportions de femelles ovulées diffèrent significativement $(P<0.05)$ entre les groupes $5^{\circ} \mathrm{C}$ $\mathrm{JC}$ et $5^{\circ} \mathrm{C} \mathrm{JL}$. À $10^{\circ} \mathrm{C}$, les niveaux de GTH II ne présentent aucune élévation quelle que soit la photopériode (fig. 5). Aucune ovulation n'est enregistrée à cette température. $\dot{A} 5^{\circ} \mathrm{C}$, chez les femelles en cours d'ovulation, la GTH II plasmatique augmente significativement $(P<0.01)$ par rapport au niveau de base mesuré le 23/01. L'augmentation de la GTH II plasmatique au cours des semaines qui précèdent et surtout qui suivent l'ovulation est plus marquée $(P<0.05)$ chez les femelles transférées en JC que chez celles qui sont maintenues en JL (fig. 6).

La GTH I plasmatique demeure continuellement à des niveaux très faibles chez tous les poissons, quelles que soient la photopériode et la température. Les niveaux de GTH l et de GTH Il chez les femelles ayant reçu des implants de mélatonine ne diffèrent pas de ceux des femelles non traitées à la mélatonine, quelles que soient la photopériode et la tempërature (résultats non présentés).

Dans tous les groupes traités au GnRH le 28/02, la GTH II augmente significativement 24 heures après le traitement. $\dot{A} 5^{\circ} \mathrm{C}$, l'adjonction de pimozide au $\mathrm{GnRH}$ 

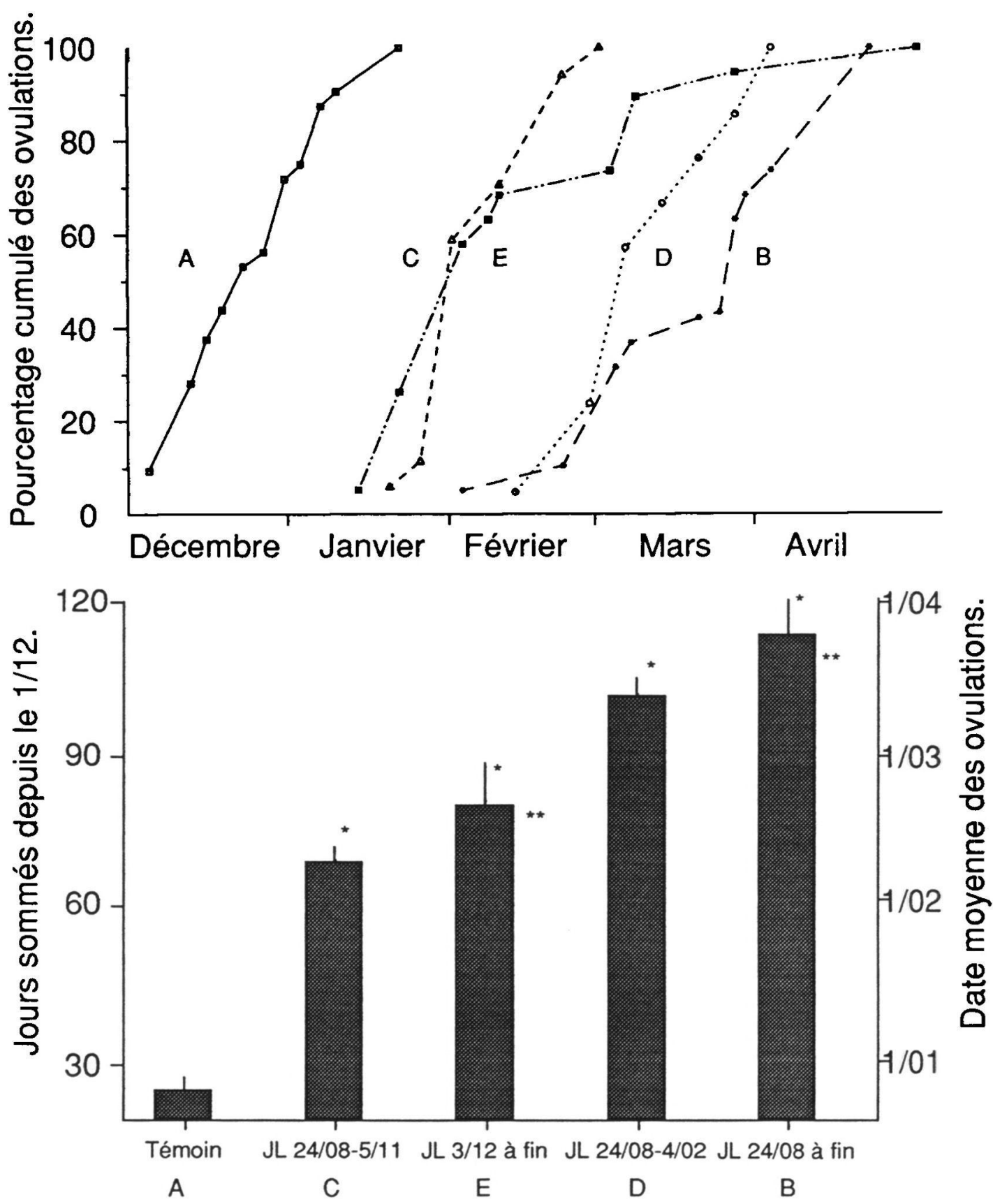

Figure 3

Effets de différentes périodes de conditionnement en jours longs sur les rythmes d'ovulation chez l'omble chevalier. Les dates moyennes des ovulations sont calculées en attribuant à chaque ovulation un nombre de jours correspondant à la somme des jours écoulés depuis le 1/12. * date moyenne d'ovulation significativement retardée par rapport aux témoins $(P<0.01)$. ${ }^{\star \star}$ variance de la date moyenne des ovulations significativement supérieure à celle des témoins $(P<0.05)$.

Figure 3

Effects of different patterns of acclimatization in long days on ovulation timing in Arctic charr. Mean time to ovulation is calculated by the mean of the number of elapsed days from 1 December to the date of ovulation for each female. * mean time to ovulation significantly delayed in comparison with controls $(P<0.01)$. ${ }^{\star \star \star}$ variance of mean time to ovulation significantly higher than in controls. 

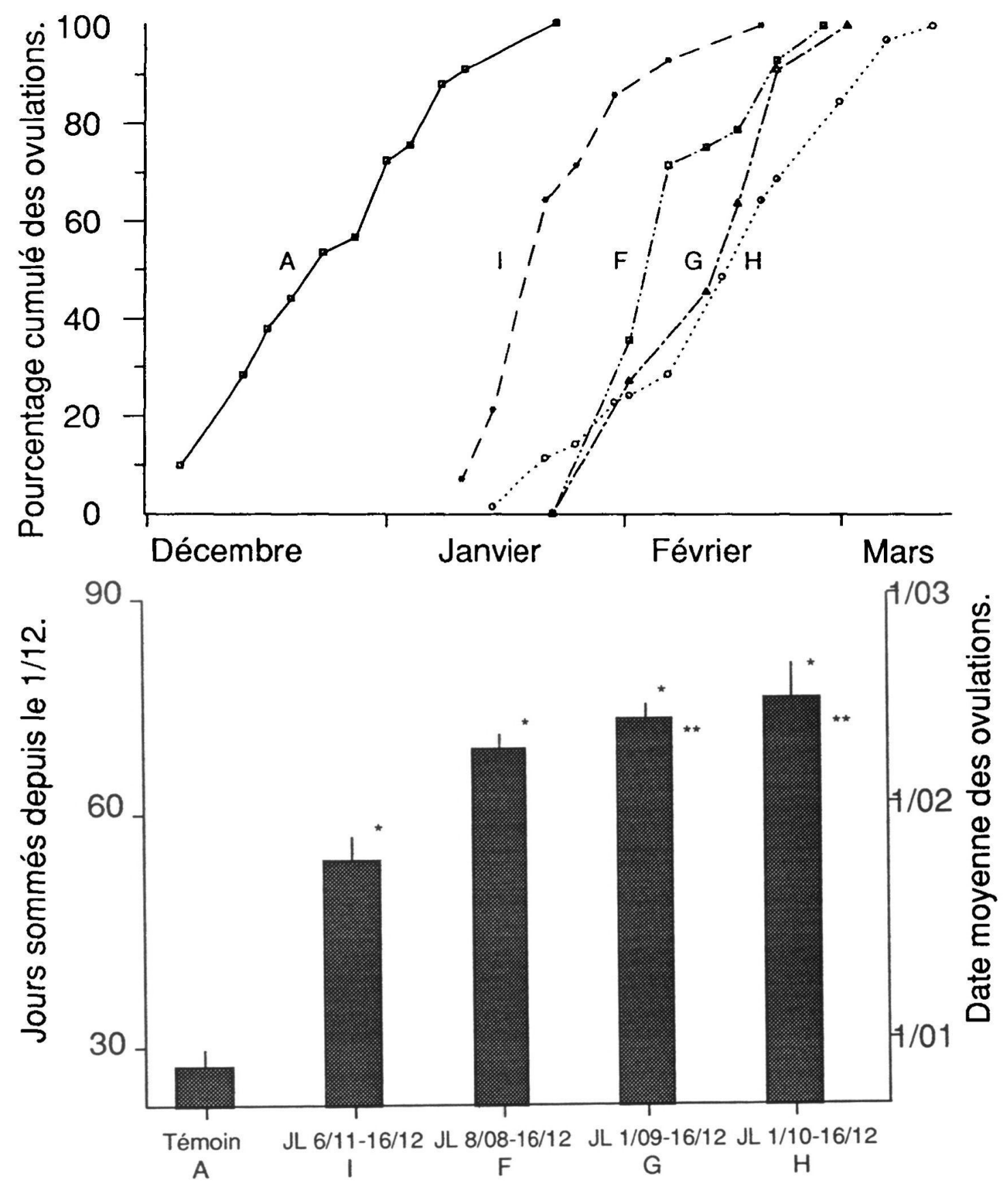

Figure 4

Mêmes légendes que la figure 2. *^ date moyenne d'ovulation significativement différente de celle du groupe 1 .

Figure 4

Same legends as in figure 2 . $^{\star \star}$ mean date to ovulation significantly different from group I $(P<0.01)$. 


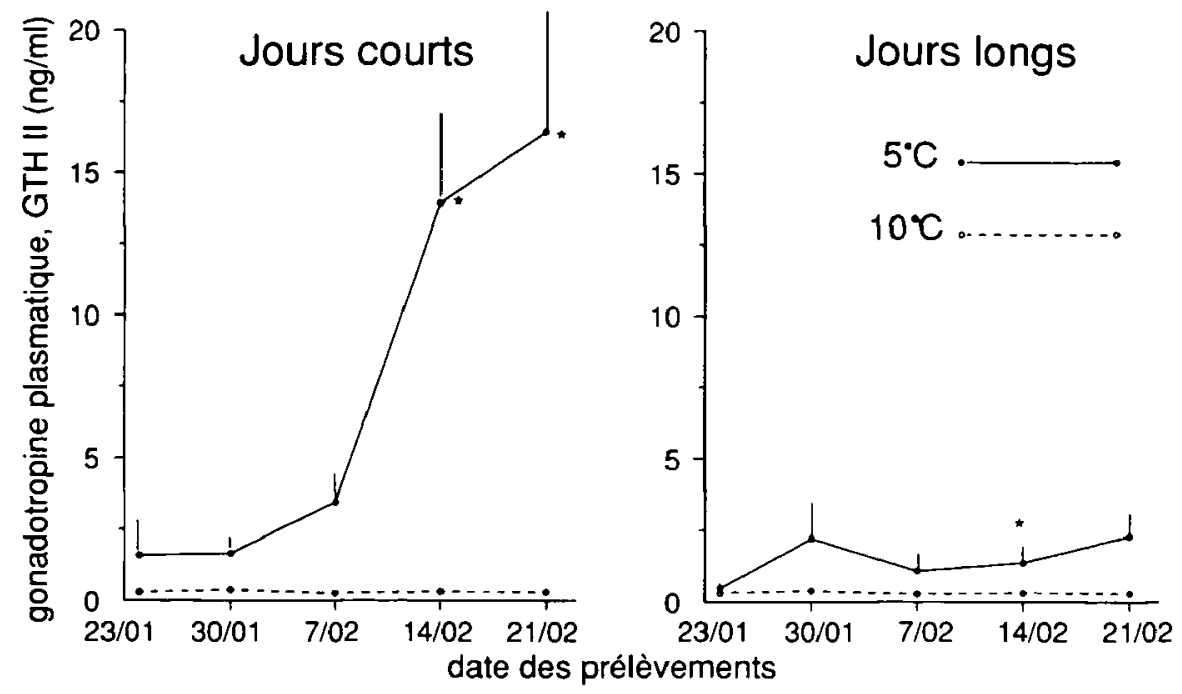

Figure 5

Évolution de la gonadotropine plasmatique (GTH II) après un transfert en jours courts ou un maintien en jours longs à 5 et $10^{\circ} \mathrm{C}$, les $23 / 01 .{ }^{*}: P<0.05$ par rapport au premier prélèvement.

\section{Figure 5}

Evolution of plasma gonadotropin (GTH II) after a transfer to short days or an exposure to long days at 5 and $10^{\circ} \mathrm{C}$, on 23 January. ${ }^{*}: P<0.05 /$ first sample on 23 January.

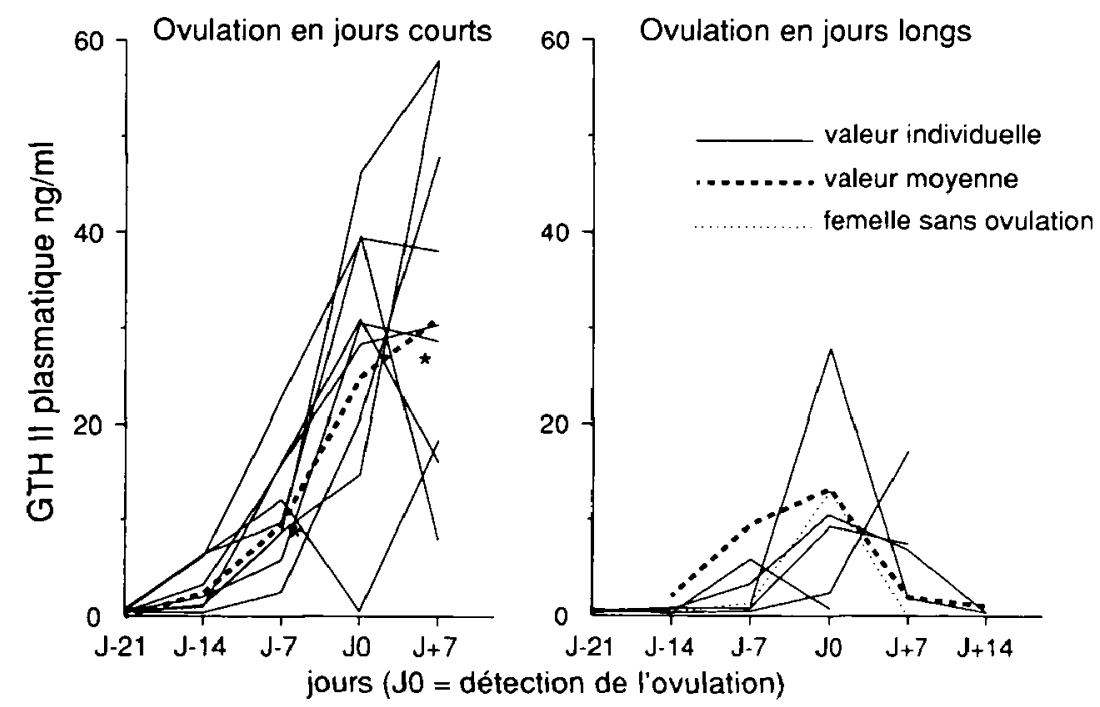

Figure 6

Évolution de la GTH II dans le plasma des femelles au cours de l'ovulation à $5^{\circ} \mathrm{C}$, en jours courts et en jours longs. La courbe en pointillés en jours longs représente un pic de GTH II chez une femelle qui ovulera un mois plus tard. ${ }^{\star}: P<0.05$ entre les valeurs en JC et en JL.

\section{Figure 6}

Evolution of plasma gonadotropin (GTH II) around the period of ovulation at $5^{\circ} \mathrm{C}$ under short days or long days. The dotted curve represents an increase of plasma GTH II in a female which will ovulate one month later. ${ }^{\star}: P<0.05$ between GTH II values in short day and long day conditions. 
ne se traduit pas par une amplification de la décharge gonadotrope par rapport à celle observée avec le $\mathrm{GnRH}$ seul, quelle que soit la photopériode (fig. 7, A et $\mathrm{B}$ ). $\dot{A} 10^{\circ} \mathrm{C}$, un traitement associant le $\mathrm{GnRH}$ et le pimozide stimule plus fortement la sécrétion gonadotrope que le $\mathrm{GnRH}$ seul, quelle que soit la photopériode (fig. 7, $\mathrm{C}$ et $\mathrm{D}$ ).

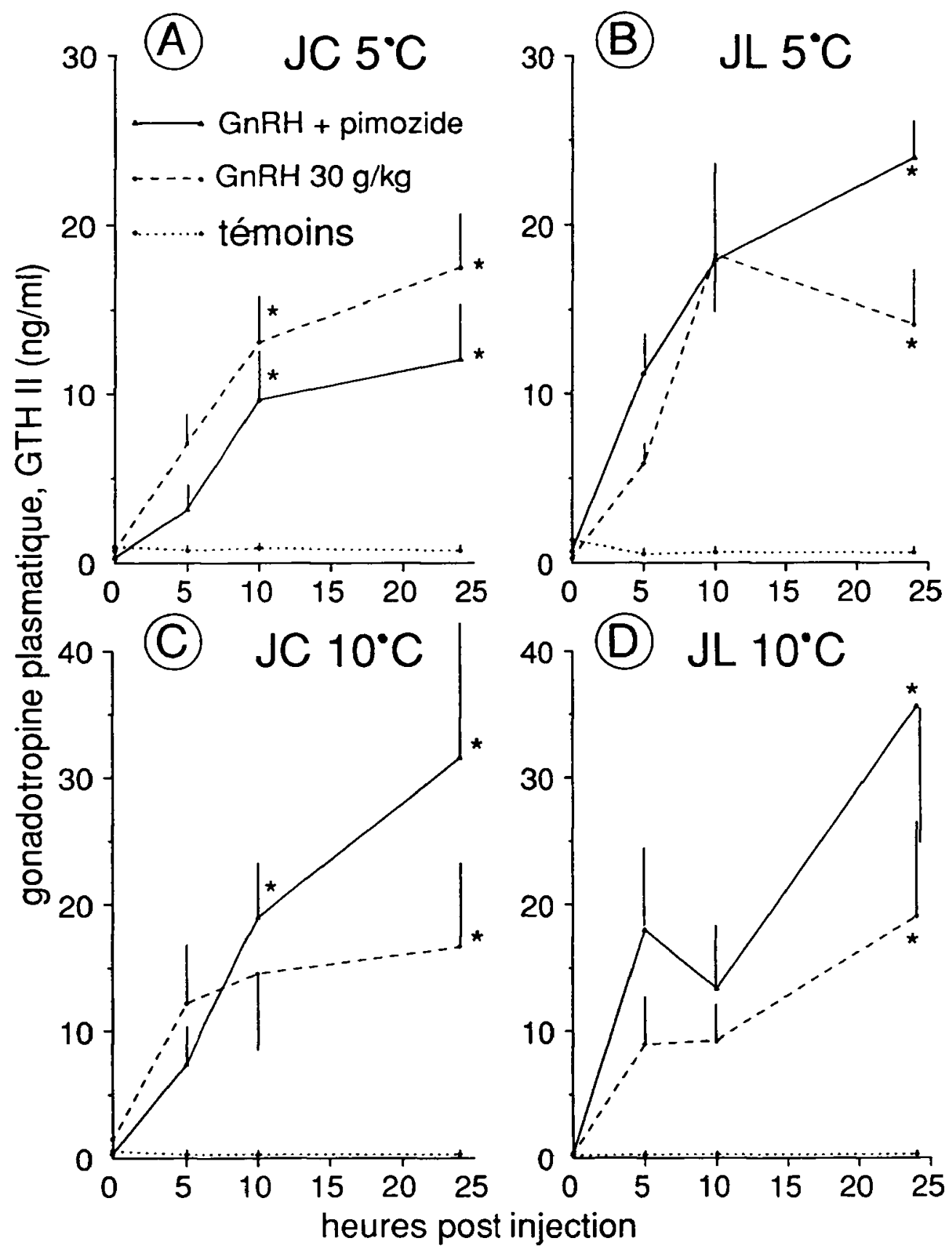

Figure 7

Stimulation de la sécrétion de la GTH II plasmatique par une injection de D Arg ${ }^{6}$ sGnRH seul ou associé au pimozide, en jours courts et en jours longs, à 5 et $10^{\circ} \mathrm{C} .{ }^{*}: \mathrm{P}<0.05$ par rapport au premier prélèvement.

Figure 7

Stimulation of GTH II secretion by an injection of D Arg ${ }^{6}$ sGnRH with or without pimozide, under short days or long days, at 5 or $10^{\circ} \mathrm{C}$. ${ }^{*}: \mathrm{P}<0.05 /$ first sample. 


\section{DISCUSSION}

\section{Effet du conditionnement en jours longs sur le décalage de la période de reproduction}

Le maintien des ombles chevaliers en jours longs en automne ou en hiver retarde significativement la période de ponte comme cela a déjà été rapporté pour d'autres salmonidés (BOURLIER et BILLARD, 1984 ; BROMAGE et al., 1984). Un conditionnement en JL appliqué du 24/08 au 5/11, c'est-à-dire pendant la période de développement des ovaires provoque sensiblement le même retard de ponte qu'un traitement en JL commençant le $3 / 12$, c'est-à-dire lorsque la vitellogenèse est terminée. L'efficacité du traitement en jours longs ne semble donc pas dépendre de la date de son application, ni de sa durée, pourvu que celle-ci dépasse 1,5 mois. En effet, un conditionnement en JL du 8/08 au $17 / 12$ a sensiblement les mêmes effets qu'un conditionnement commencé un ou deux mois plus tard. Toutefois, ces traitements sont plus efficaces qu'un traitement en JL allant du 6/11 au 16/12. Ces résultats peuvent s'expliquer au moins en partie en formulant l'hypothèse qu'il faudrait un conditionnement d'une durée de quelques semaines en $\mathrm{JL}$ en fin de cycle reproducteur pour qu'une inhibition de la sécrétion gonadotrope de type II se mette progressivement en place. Cette inhibition atteindrait sa valeur maximale après un mois et demi environ de conditionnement en JL. Le reconditionnement des poissons en JC permettrait la levée de cette inhibition en l'espace de quelques semaines, ce qui permettrait d'obtenir des ovulations relativement bien regroupées lorsque les poissons ne sont pas maintenus en JL pendant la ponte (GILLET, 1994). Chez les ovins, le déclenchement de l'activité ovulatoire après un transfert en JC de même que l'inhibition de cette activité après un reconditionnement en $\mathrm{J} L$ se produisent avec un décalage d'un mois environ par rapport au changement de régime photopériodique (MALPAUX et al., 1996). Chez ces animaux, la mélatonine sert de relais entre l'information photopériodique et les centres nerveux contrôlant les sécrétions gonadotropes. La mélatonine agit en modifiant des liaisons nerveuses, ce qui explique cette latence (MALPAUX et al., 1996). II est possible qu'un mécanisme analogue puisse exister chez l'omble chevalier.

\section{Effet d'un transfert en jours longs en fin de cycle reproducteur sur les sécrétions gonadotropes}

Chez les salmonidés, les niveaux de la GTH II sont indétectables en cours de vitellogenèse (SWANSON, 1991). Ils ne commencent à augmenter que quelques jours avant la maturation ovocytaire (BRETON et al., 1997a) et il se produit une décharge maturante de quelques heures juste avant ce phénomène (PRAT et al., 1996 ; BRETON et al., 1997a). Une augmentation comparable se produit chez l'omble chevalier (BRETON et al., 1997b). Ainsi, à la fin du cycle reproducteur, le maintien prolongé des femelles sous un régime de $J L$ s'accompagne d'une forte inhibition de la sécrétion gonadotrope (GTH II). En effet, les niveaux plasmatiques de la GTH II sont très faibles $(0.5 \mathrm{ng} / \mathrm{ml})$ le 23/01 chez les poissons conditionnés depuis plus de quatre mois en $\mathrm{JL}$. Cette inhibition de la sécrétion gonadotrope est réversible car cette hormone augmente en l'espace de quelques semaines dans le plasma des animaux retransférés en JC. Ces observations sont en conformité avec l'hypothèse formulée dans le paragraphe précédent. La sensibilité hypophysaire à une stimulation de la sécrétion gonadotrope sous l'effet d'une injection de GnRH exogène n'est pas sensiblement diminuée par le conditionnement en $\mathrm{JL}$. En réponse à un traitement au D Arg $^{6}$ sGnRH, les niveaux de la GTH II plasmatique augmentent de façon pratiquement identique sous les deux régimes photopériodiques testés. Le pimozide, antagoniste de la dopamine, ne potentialise pas l'effet du GnRH chez les poissons en JL. Cela signifie qu'il existe probablement une différence entre les mécanismes d'inhibition de la sécrétion gonadotrope mis en place en fin de cycle reproducteur en réponse à un conditionnement en $\mathrm{JL}$ ou en réponse à une acclimatation à une température trop élevée qui se traduit par l'apparition d'une inhibition de type dopaminergique de l'action du GnRH (BRETON et al., 
1997b). Cependant, le conditionnement des femelles en $J L$ ne provoque pas l'inhibition totale des ovulations comme cela s'observe après une acclimatation à $10^{\circ} \mathrm{C}$ en automne (GILLET et al., 1996). Les femelles maintenues en JL parviennent à ovuler, bien que le rythme des ovulations soit très ralenti par rapport aux poissons en JC. Dans ce cas, la décharge ovulante de GTH II est de plus faible amplitude que chez les femelles gardées en JC. Sa durée est également plus faible. Ainsi, parallèlement à une inhibition de la sécrétion basale de la GTH II, l'action des jours longs semble aussi se traduire sur la sécrétion tonique de la GTH II, par un mécanisme indéterminé ne mettant pas en jeu la réceptivité hypophysaire.

Les niveaux de la GTH I plasmatique restent inchangés quelles que soient la température et la photopériode. Cette absence de sensibilité de la GTH I qui contrôle la vitellogenèse (SWANSON, 1991), à ces facteurs externes pourrait expliquer pourquoi le développement des ovaires s'achève quel que soit le conditionnement des géniteurs. La mélatonine administrée en continu sous forme d'implant ne modifie pas la sécrétion gonadotrope des poissons quelle que soit la photopériode testée. Ceci ne permet pas d'exclure un rôle possible de la mélatonine dans le transfert de l'information photopériodique vers les structures nerveuses contrôlant la sécrétion gonadotrope. En effet, d'une part, la sécrétion de la mélatonine n'augmente que pendant la nuit en conditions naturelles et, d'autre part, l'effet stimulateur des JC sur la sécrétion gonadotrope n'a été reproduit par des implants de mélatonine que chez les ovins et les caprins (MALPAUX et al., 1996). Les recherches sur la photopériode chez les salmonidés ont surtout concerné la truite arc-en-ciel, en raison de son importance économique. Chez cette espèce, d'une part, aucun traitement photopériodique ne permet d'inhiber totalement la reproduction et, d'autre part, le cycle reproducteur évolue suivant un rythme circannuel en condition d'éclairement quotidien constant. Ces observations ont conduit certains auteurs à formuler l'hypothèse que la photopériode n'agirait qu'en entraînant un rythme interne responsable du déroulement du cycle reproducteur et en synchronisant les individus (DUSTON et BROMAGE, 1988). Chez l'omble chevalier, nos résultats confirment qu'aucun traitement photopériodique ne permet d'inhiber totalement la reproduction. En fin de cycle reproducteur, il est probable cependant que la photopériode exerce un contrôle passant par une inhibition partielle de la sécrétion de la GTH II. Ce contrôle pourrait impliquer les mécanismes responsables de la libération du $\mathrm{G} n \mathrm{RH}$ et présenterait des similitudes avec ce qui a été décrit chez les ovins (MALPAUX et al., 1996).

\section{BIBLIOGRAPHIE}

BOURLIER A., BILLARD R., 1984. Delayed gametogenesis and spawning in rainbow trout (Salmo gairdneri) kept under permanent light during the first and second reproductive cycle. Can. J. Zool., 62, 2183-2187.

BRETON B., MAISSE G., LEMENN F., 1983. Contrôle photopériodique de la saison de reproduction en salmoniculture : une expérience pilote en Bretagne. Bull. Fr. Piscic., 288, 35-45.

BRETON B., GOVORUM M., MIKOLAJCZYK T., 1997a. GTH I and GTH II secretion profiles during the reproductive cycle in female rainbow trout, relationship with pituitary responsiveness to GnRH-A stimulation. Gen. Comp. Endocrinol. (in press).

BRETON B., GILLET C., JALABERT B., 1997b. Relations entre température, blocage de la maturation et axe gonadotrope chez l'omble chevalier (Salvelinus alpinus). Gen. Comp. Endocrinol. (in press).

BROMAGE N.R., ELLIOT J.A., SPRINGATE J.C., WHITEHEAD C., 1984. The effects of constant photoperiods on the timing of spawning in the rainbow trout. Aquaculture, 48, 213-223. 
BROMAGE N.R., DUSTON J., 1986. The control of spawning in the rainbow trout (Salmo gairdneri Richardson) using photoperiod techniques. Inst. Freshwater Res. drottningholm Rep., 63, 26-35.

DUSTON J., BROMAGE N.R., 1988. The entrainment and gating of the endogenous circannual rhythm of reproduction in the female rainbow trout (Salmo gairdneri). J. Comp. Physiol., A 164, 259-268.

GILLET C., 1991. Egg production in an Arctic charr (Salvelinus alpinus) broodstock : effects of temperature on the timing of spawning and the quality of eggs. Aquat. Living Resour., 4, 109-116.

GILLET C., 1994. Egg production in an Arctic charr (Salvelinus alpinus) broodstock : effects of photoperiod on the timing of ovulation and egg quality. Can. J. Zool., 72, 334-338.

GILLET C., BRETON B., MIKOLAJCZYK T., 1996. Effects of GnRHa and pimozide treatments on the timing of ovulation and on egg quality in Arctic charr (Salvelinus alpinus) at 5 and $10^{\circ} \mathrm{C}$. Aquat. Living Resour., 9, 257-263.

GOVORUM M., BRETON B., 1997. Immunological cross reactivity between rainbow trout GTH I and GTH II and their a and b subunits : application to the development of homologous radioimmuno assays. Gen. Comp. Endocrinol. (in press).

MAISSE G., BRETON B., 1996. Contrôle photopériodique de la saison de reproduction chez les salmonidés. INRA Productions animales, 9, 71-77.

MALPAUX B., VIGUIÉ C., THIERY J.C., CHEMINEAU P., 1996. Contrôle photopériodique de la reproduction. INRA Productions animales, 9, 9-23.

PRAT F., SUMPTER J.P., TYLER C.A., 1996. Validation of radioimmuno assays for two salmon gonadotropins (GTH I and GTH II) and their plasma concentrations throughout the reproductive cycle in male and female rainbow trout (Onchorhynchus mykiss). Biol. Reprod., 54, 1375-1382.

SWANSON P., 1991. Salmon gonadotropin reconciling old and new ideas. In Scott A.P., Sumpter J.P., Kime D.K. and Rolfe M.S. (eds.), Proc. 4" Int. Symp. on the reproductive physiology of fish, 2-7. Fish Symposium 1991, Sheffield. 